\title{
Observation of Spin-Valley-Coupling-Induced Large Spin-Lifetime Anisotropy in Bilayer Graphene
}

\author{
Johannes Christian Leutenantsmeyer, ${ }^{1, * \dagger}$ Josep Ingla-Aynés, ${ }^{1, *}$ Jaroslav Fabian, ${ }^{2}$ and Bart J. van Wees ${ }^{1}$ \\ ${ }^{1}$ Physics of Nanodevices, Zernike Institute for Advanced Materials, University of Groningen, 9747 AG Groningen, Netherlands \\ ${ }^{2}$ Institute for Theoretical Physics, University of Regensburg, 93040 Regensburg, Germany
}

(Received 2 May 2018; published 20 September 2018)

\begin{abstract}
We report the first observation of a large spin-lifetime anisotropy in bilayer graphene (BLG) fully encapsulated between hexagonal boron nitride. We characterize the out-of-plane $\left(\tau_{\perp}\right)$ and in-plane $\left(\tau_{\|}\right)$spin lifetimes by oblique Hanle spin precession. At $75 \mathrm{~K}$ and the charge neutrality point (CNP), we observe a strong anisotropy of $\tau_{\perp} / \tau_{\|}=8 \pm 2$. This value is comparable to graphene-transition-metal-dichalcogenide heterostructures, whereas our high-quality BLG provides with $\tau_{\perp}$ up to $9 \mathrm{~ns}$, a spin lifetime more than 2 orders of magnitude larger. The anisotropy decreases to $3.5 \pm 1$ at a carrier density of $n=6 \times 10^{11} \mathrm{~cm}^{-2}$. Temperature-dependent measurements show above $75 \mathrm{~K}$ a decrease of $\tau_{\perp} / \tau_{\|}$with increasing temperature, reaching the isotropic case close to room temperature. We explain our findings with electric-field-induced spin-valley coupling arising from the small intrinsic spin-orbit fields in BLG of $12 \mu \mathrm{eV}$ at the CNP.
\end{abstract}

DOI: 10.1103/PhysRevLett.121.127702

Coupling between the electronic spin and valley degree of freedom arises in materials without inversion symmetry such as single layer transition-metal-dichalcogenides (TMDs) [1,2] where the electronic bands are spin split by the spin-orbit fields. Because of time-reversal symmetry, the induced spin splitting is opposite for the $K$ and $K^{\prime}$ points of the Brillouin zone. This leads to a coupling between the spin and valley degrees of freedom and enables new functionalities such as the optical injection of spin currents with circularly polarized light $[3,4]$. The spinvalley coupling has been imprinted on the band structure of monolayer graphene by placing it in proximity with a transition-metal dichalcogenide (TMD) and measured using spin [5-7] and charge transport [8-10]. However, it remains a question if similar behavior can be observed in pristine graphene devices.

Bilayer graphene (BLG) has an intrinsic spin-orbit coupling (SOC) of $\lambda_{I} \sim 12 \mu \mathrm{eV}$, which points out the BLG plane. A perpendicular electric field induced by asymmetric crystal alignment, gating, and/or doping breaks the inversion symmetry, and, as a consequence, the intrinsic SOC induces an out-of-plane spin splitting of $2 \lambda_{I} \sim 24 \mu \mathrm{eV}$ at the $K$ points [11]. The splitting has opposite sign in $K$ and $K^{\prime}$ and, therefore, a valley dependence. Recent ab initio calculations show that the encapsulation of BLG in hexagonal boron nitride $(h$-BN) preserves the presence of the spin splitting with a similar magnitude [12].

Thermal broadening and inhomogeneities due to doping fluctuations [13] prevent the direct measurement of such a small spin splitting in conventional charge transport experiments. However, spin precession experiments can resolve spin splittings much smaller than $k_{B} T$ if the splitting extends over a sufficiently large region in reciprocal space and energy [14]. In the presence of an out-of-plane spin splitting, the dephasing of spins follows the Dyakonov-Perel mechanism [15]. The in-plane spin lifetime $\tau_{\|}$is inversely proportional to the intervalley scattering time, $\tau_{\|} \propto \lambda_{I}^{2} / \tau_{\text {iv }}$ [5]. Hence, $\tau_{\|}$is sensitive to the SOC strength.

Apart from the intrinsic SOC, breaking of the inversion symmetry leads to Rashba spin-orbit fields in the graphene plane $[16,17]$ that affect both in-plane and out-of-plane $\left(\tau_{\perp}\right)$ spin lifetimes. Therefore, spin relaxation in BLG is a result of an interplay between intrinsic and Rashba SOC. The Rashba SOC depends on the Fermi velocity, which increases with the carrier density $n$, whereas the intrinsic spin-orbit splitting decreases with $n$. As a consequence, the spin-lifetime anisotropy $\left(\tau_{\perp} / \tau_{\|}\right)$is expected to depend strongly on $n$ near the CNP $[11,18]$, allowing the electrical control of the spin-lifetime anisotropy.

Here, we study $\tau_{\perp}$ and $\tau_{\|}$in fully $h$-BN encapsulated BLG using oblique spin precession. Our results show that in contrast with monolayer graphene [17,19-21], at temperatures below $300 \mathrm{~K}$, the ratio $\tau_{\perp} / \tau_{\|}$is significantly above 1 over the full measured range of $n$. At $75 \mathrm{~K}$, we observe a dependence of $\tau_{\perp} / \tau_{\|}$on the carrier concentration, which increases from $3.5 \pm 1$ at $n=6 \times 10^{11} \mathrm{~cm}^{-2}$ to $8 \pm 2$ at the CNP, confirming the role of the spin-valley coupling on the spin transport. The anisotropy at the CNP is comparable to graphene-TMD systems $[6,7]$. However, the spin lifetimes in our BLG devices are 2 orders of magnitude larger [22-27]. These results show that small spin-orbit fields can induce sizable effects on the spin relaxation and indicate 
(a)

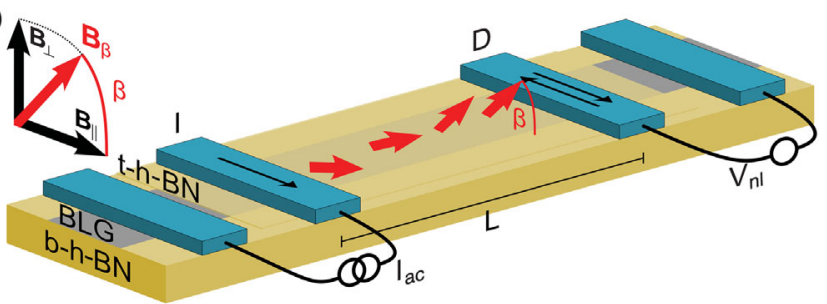

(b)

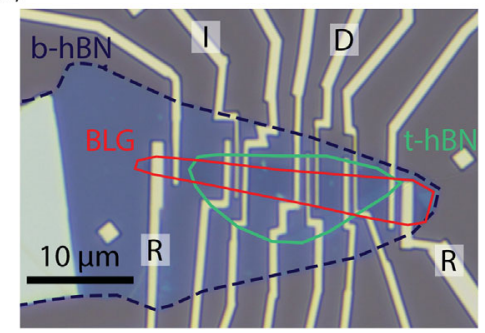

(c)

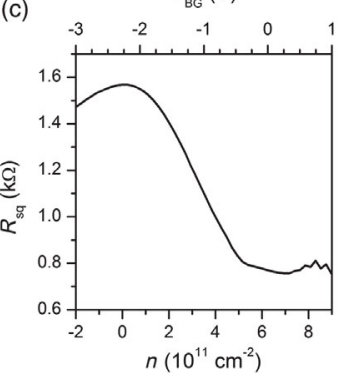

FIG. 1. (a) Schematic and (b) optical image of the device geometry. BLG is encapsulated by a $1 \mathrm{~nm}$ thick $h$-BN tunnel barrier $(t-h-\mathrm{BN})$ and a $5 \mathrm{~nm}$ bottom $h$-BN flake $(b-h$-BN). A lowfrequency ac current $\left(I_{\mathrm{ac}}\right)$ injects a spin accumulation into the BLG. The nonlocal signal $\left(V_{\mathrm{nl}}\right)$ is measured using standard lockin technique. The precession of injected in-plane spins around the magnetic field $\left(B_{\beta}\right)$ is illustrated in the encapsulated BLG channel. Note that the outer reference contacts $(R)$ are not covered by the $h$-BN tunnel barrier. The injector $(I)$ and detector contact $(D)$ used for the measurements discussed in the main text are labeled and have a spacing of $L=7 \mu \mathrm{m}$. (c) Gate voltage and carrier concentration dependence of the BLG square resistance.

that the spin relaxation in our devices is limited by $\lambda_{I}$ and the Rashba SOC.

The device is shown in Fig. 1 where the BLG is protected from contamination by a trilayer $h$ - BN tunnel barrier on top and a $5 \mathrm{~nm}$ thick bottom $h$-BN flake below [28]. The stack is deposited on a $90 \mathrm{~nm} \mathrm{SiO}_{2} / \mathrm{Si}$ wafer which is used as a back gate. Ferromagnetic cobalt contacts are defined using standard electron-beam lithography and election-beam evaporation techniques and are used for spin injection and detection. The contacts are noninvasive with a resistancearea product of $2 \mathrm{M} \Omega \mu \mathrm{m}^{2}$. With a back gate, we tune the carrier concentration from the hole regime, slightly beyond the CNP $\left(2 \times 10^{11} \mathrm{~cm}^{-2}\right)$ up to $6 \times 10^{11} \mathrm{~cm}^{-2}$ in the electron regime. The CNP is at $V_{\mathrm{BG}}=-2 \mathrm{~V}$ applied to the back gate, indicating a small background doping. The electric field at the CNP is estimated to be between 40 and $80 \mathrm{mV} / \mathrm{nm}$ [29]. Note that the application of large electric fields (above $2 \mathrm{~V} / \mathrm{nm}$ ) to BLG can result in band gaps up to $200 \mathrm{meV}$ [32-34]. However, the small fields applied to our sample lead to band gap openings significantly smaller than $k_{B} T$ and are neglected in our analysis.

The mobility $\mu$ of the sample is $12000 \mathrm{~cm}^{2} / \mathrm{Vs}$ at $n=4 \times 10^{11} \mathrm{~cm}^{-2}$ obtained using $\mu=(1 / e)(d \sigma / d n)$ where $\sigma$ is the conductivity and $e$ the electron charge. The charge diffusion coefficient is $D_{c}=0.026 \mathrm{~m}^{2} / \mathrm{s}$,

which is in agreement with the spin diffusion coefficient $D_{s}=(0.021 \pm 0.005) \mathrm{m}^{2} / \mathrm{s}$ obtained from Hanle spin precession. This indicates the consistency of the analysis.

To optimize the spin injection efficiency, we apply additionally to the ac measurement current a dc bias current of $-0.6 \mu \mathrm{A}$ to the trilayer $h$-BN barrier $[35,36]$. Note that the negative bias applied to the injector causes a sign change in the spin polarization of the injector and, therefore, in $R_{\mathrm{nl}}$. For comparison with conventional Hanle curves, we have inverted the sign of $R_{\mathrm{nl}}$ (see Ref. [29]).

Figures 2(a)-2(c) show the experimental results obtained from oblique Hanle spin precession [see Fig. 1(a) for the schematics of the measurement] at three different carrier densities. The data shown in Figs. 2(a) and 2(d) are measured at $n=6 \times 10^{11} \mathrm{~cm}^{-2}$ and Figs. 2(b) and 2(e) at $n=4 \times 10^{11} \mathrm{~cm}^{-2}$, whereas the data in Figs. 2(c) and 2(f) are measured at the CNP. $R_{\mathrm{nl} \beta}$ is defined as the spin signal where the spin accumulation perpendicular to the magnetic field $B_{\beta}$ is fully dephased. We extract $R_{\mathrm{n} 1 \beta}$ from the experiment by averaging $R_{\mathrm{nl}}$ between 50 and $100 \mathrm{mT}$ indicated by the gray area at low magnetic fields in Figs. 2(a)-2(c).

The spins are injected collinear to the in-plane magnetization of the ferromagnetic electrode with efficiency $P$. Since only the component parallel to $B_{\beta}$ is conserved, the injection and detection efficiencies for the measured spins become $P \times \cos (\beta)$. Consequently, $R_{\mathrm{nl} \beta}$ is proportional to $\cos ^{2}(\beta)$. Therefore, at $\beta=45^{\circ}$, one would expect $R_{\mathrm{nl} \beta}$ to be reduced by $50 \%$ compared to $R_{\mathrm{nl} 0}$ in an isotropic system. We find at $75 \mathrm{~K}$ that at all different carrier concentrations in Figs. 2(a)-2(c), $R_{\mathrm{nl} \beta} / R_{\mathrm{nl} 0}$ is clearly above 0.5 for $\beta=45^{\circ}$, which can only be the case if $\tau_{\perp} / \tau_{\|}>1$. This can be seen from Eq. (2), which can be used to quantify the degree of anisotropy $[20,37]$ :

$$
\begin{aligned}
\frac{R_{\mathrm{nl} \beta}}{R_{\mathrm{nl} 0}} & =\sqrt{\frac{\tau_{\beta}}{\tau_{\|}}} \exp \left[\frac{-L}{\lambda_{\|}}\left(\sqrt{\frac{\tau_{\|}}{\tau_{\beta}}}-1\right)\right] \cos ^{2}(\beta), \\
\frac{\tau_{\beta}}{\tau_{\|}} & =\left(\cos ^{2}(\beta)+\frac{\tau_{\|}}{\tau_{\perp}} \sin ^{2}(\beta)\right)^{-1} .
\end{aligned}
$$

However, this model is only applicable for a channel significantly longer than both the in-plane and out-of-plane spin relaxation lengths. The out-of-plane spin relaxation length $(\sim 12 \mu \mathrm{m})$ is longer than the closest spacing between the sample edge and the injector $(8 \mu \mathrm{m})$. Therefore, the exact device geometry has to be taken into account for a quantitative analysis.

To carefully account for the device geometry, we solve the Bloch equations for anisotropic spin transport numerically. Furthermore, we include both the effect of $B_{\beta}$ on the contact magnetization direction using a Stoner-Wohlfarth model and the influence of the finite resistances of the reference contacts $[29,38,39]$. The Hanle precession curves 

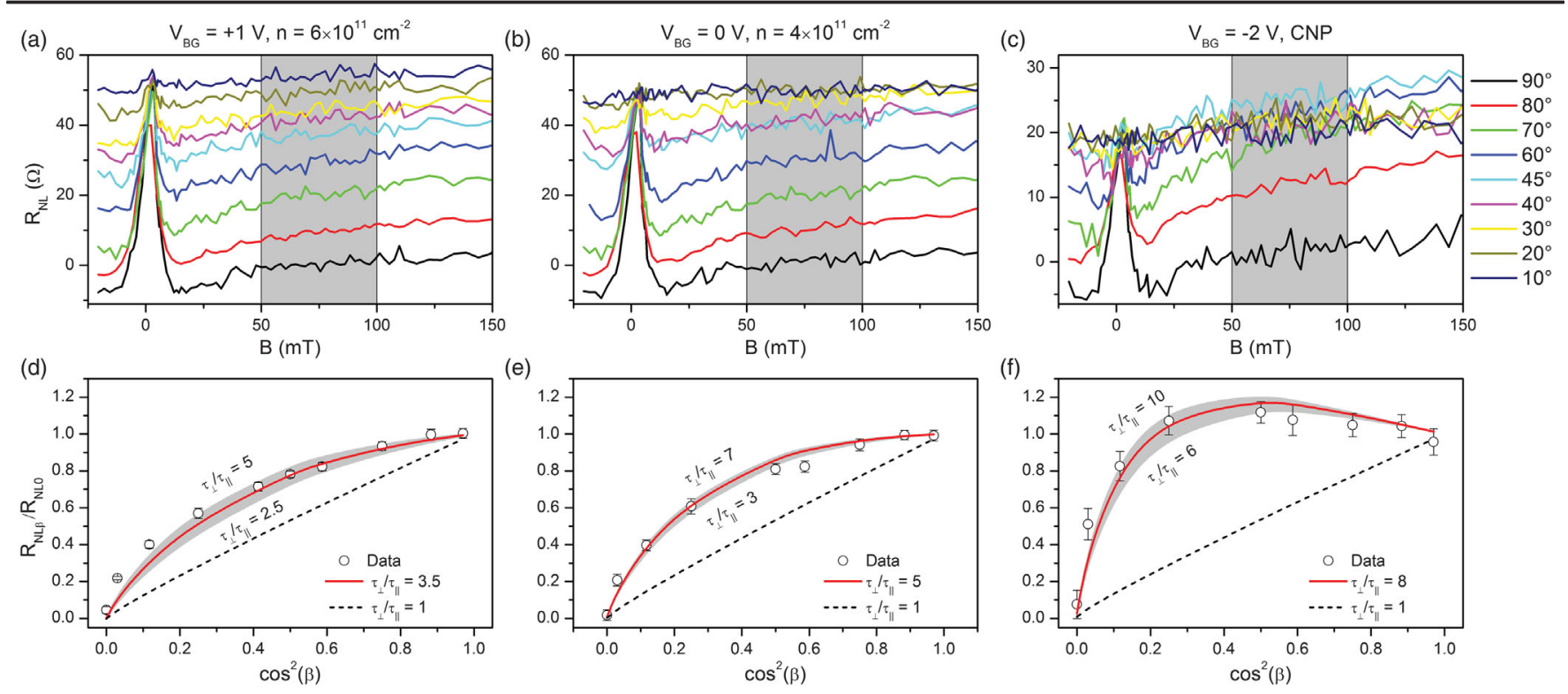

FIG. 2. Oblique Hanle spin precession data for (a) $n=6 \times 10^{11} \mathrm{~cm}^{-2}$, (b) $n=4 \times 10^{11} \mathrm{~cm}^{-2}$, and (c) the charge neutrality point (CNP). $R_{\mathrm{nl} 0}$ denotes the nonlocal resistance at zero field and $R_{\mathrm{n} \mid \beta}$ the nonlocal resistance where the perpendicular spin component has fully dephased. $R_{\mathrm{nl} \beta}$ is obtained by averaging $R_{\mathrm{nl}}$ over the shaded area (50-100 $\mathrm{mT}$ ). The bottom panels (d)-(f) show the comparison between the ratios $R_{\mathrm{n} 1 \beta} / R_{\mathrm{n} 10}$ and our model for different anisotropy values. The shaded area corresponds to the estimated error margin with the denoted anisotropy values. Note that panels (a)-(c) have a small background in $R_{\mathrm{nl}}$ of $9.3,18$, and $17.8 \Omega$ subtracted.

are simulated for different ratios $\tau_{\perp} / \tau_{\|}$and different angles $\beta$. We obtain $R_{\mathrm{nl} \beta} / R_{\mathrm{nl0}}$ from the simulated curves using the same procedure as used for the experimental data.

The resulting curves are shown in Figs. 2(d)-2(f) where the red solid line represents the best fit to the data. The gray areas correspond to the estimated error margin with the annotated values. The case of an isotropic system is shown by the dotted gray lines. We find $\tau_{\perp} / \tau_{\|}$to be $3.5 \pm 1$ $\left(n=6 \times 10^{11} \mathrm{~cm}^{-2}\right), \quad 5 \pm 2 \quad\left(n=4 \times 10^{11} \mathrm{~cm}^{-2}\right)$, and $8 \pm 2$ (CNP). We have measured and analyzed different contact spacings and different injector or detector contact pairs, which all showed a consistent behavior and are discussed in the Supplemental Material [29].
When a large $B_{\perp}$ is applied, the Co magnetization direction rotates out of the sample plane. As a consequence, a perpendicular spin component is injected making $R_{\mathrm{nl}}$ sensitive to the spin-lifetime anisotropy [19]. The data measured up to a large $B_{\perp}$ are shown in Fig. 3 together with the simulated Hanle curves. It should be noted that for all carrier concentrations, $R_{\mathrm{nl}}\left(B_{\perp}=1.1 \mathrm{~T}\right)$ clearly exceeds $R_{\mathrm{nl}}\left(\mathrm{B}_{\perp}=0 T\right)$, which is a direct consequence of $\tau_{\perp}>\tau_{\|}$. The Hanle curves are simulated for different $\tau_{\perp} / \tau_{\|}$ratios, where the gray lines represent the isotropic case. We attribute the difference between the low- (Fig. 2) and high-field analysis (Fig. 3) to two origins. First, our simulations use a simple out-of-plane shape anisotropy
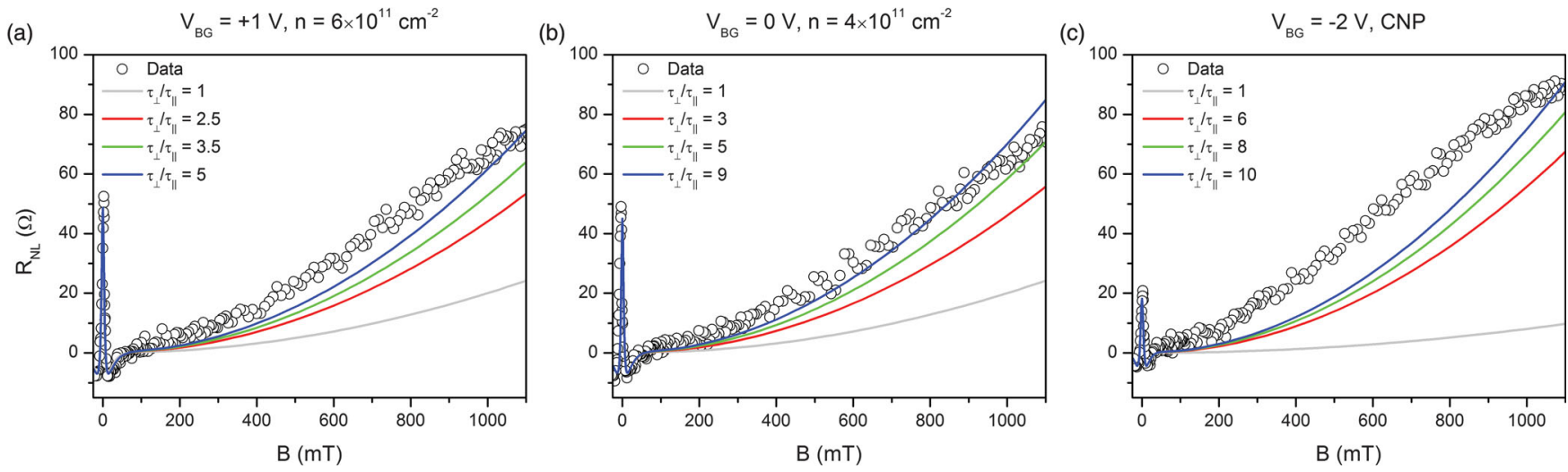

FIG. 3. High-field Hanle spin precession curves measured at $\beta=90^{\circ}$ and $T=75 \mathrm{~K}$ for (a) $n=6 \times 10^{11} \mathrm{~cm}^{-2}$, (b) $n=4 \times 10^{11} \mathrm{~cm}^{-2}$, (c) CNP. We simulate the spin precession using the parameters from Fig. 2 . The gray line corresponds to the isotropic case. The perpendicular saturation field of the cobalt contacts is $1.5 \mathrm{~T}$. Note that the same background as in Fig. 2 has been subtracted. 
model to describe the rotation of the electrode magnetizations under $B_{\perp}$, whereas the magnetization behavior can deviate from the idealized system. Second, we observe magnetoresistance of the BLG channel, which can reach up to $50 \%$ at high fields and at the CNP. Its possible influence on the measured data is discussed in the Supplemental Material [29]. However, for magnetic fields below $0.1 \mathrm{~T}$ at the CNP the magnetoresistance is below $1 \%$. Hence, magnetoresistance does not affect our low-field analysis.

We can estimate the intervalley scattering time $\tau_{\text {iv }}$ from the extracted $\tau_{\|}$and $\tau_{\perp}$ by assuming a Dyakonov-Perel type of spin relaxation as predicted theoretically $[5,15]$,

$$
\frac{1}{2 \tau_{\perp}}+\left(\frac{2 \lambda_{I}}{\hbar}\right)^{2} \tau_{\mathrm{iv}}=\frac{1}{\tau_{\|}}
$$

where $1 / \tau_{\perp}=\left(2 \lambda_{R} / \hbar\right)^{2} \tau_{p}$ with the Rashba SOC $\lambda_{R}$. The relevant spin and charge transport parameters are shown in Table I. We observe the shortest $\tau_{\text {iv }}$ at the CNP, which we attribute to two origins: First, $\lambda_{I}$ is $12 \mu \mathrm{eV}$ at the CNP but decays quickly with increasing momentum from the CNP [11]. As a consequence, the effective $\lambda_{I}$ is smaller than $12 \mu \mathrm{eV}$, and our extracted $\tau_{\text {iv }}$ should be seen as the lower bound. Second, the spin splittings have opposite sign in the conduction and valence bands. Hence, non-energyconserving scattering between both bands plays the same role as intervalley scattering when both electrons and holes contribute to the transport. $\tau_{\text {iv }}$ becomes an effective parameter $\left(\tau_{\mathrm{iv}}^{*}\right)$ determined by both intervalley and interband scatting $\left(\tau_{\mathrm{ib}}\right), \tau_{\mathrm{iv}}^{*-1}=\tau_{\mathrm{ib}}^{-1}+\tau_{\mathrm{iv}}^{-1}$.

Note that the values of $\lambda_{I}$ from Table I are calculated in pristine BLG with an applied electric field of $25 \mathrm{mV} / \mathrm{nm}$ [11]. The accurate determination of $\lambda_{I}$ from first principles requires the knowledge of the alignment between the crystal planes of $h$-BN and BLG. However, preliminary $a b$ initio calculations support the presence of a spin splitting in the range of $24 \mu \mathrm{eV}$ at the $K$ and $K^{\prime}$ points in $h$-BN encapsulated BLG under small electric fields [12].

It should be mentioned that our out-of-plane spin lifetimes in BLG (up to $9 \mathrm{~ns}$ ) are close to the largest measured

TABLE I. Spin and charge transport parameters of the discussed device. $\tau_{\text {iv }}$ is calculated using Eq. (3). The density dependence of $\lambda_{I}$ is extracted from Ref. [11] at a constant electric field of $25 \mathrm{mV} / \mathrm{nm}$. The momentum scattering time $\tau_{p}$ is obtained assuming $D_{s}=D_{c}=v_{F}^{2} \tau_{p} / 2$, where $v_{F}$ is the Fermi velocity.

\begin{tabular}{lccccccccc}
\hline \hline $\begin{array}{l}T \\
(\mathrm{~K})\end{array}$ & $\begin{array}{c}V_{\mathrm{BG}} \\
(\mathrm{V})\end{array}$ & $\begin{array}{c}D_{s} \\
\left(\mathrm{~m}^{2} / \mathrm{s}\right)\end{array}$ & $\begin{array}{c}\tau_{\|} \\
(\mathrm{ns})\end{array}$ & $\begin{array}{c}\tau_{\perp} \\
(\mathrm{ns})\end{array}$ & $\tau_{\perp} / \tau_{\|}(\mu \mathrm{eV})$ & $\begin{array}{c}\lambda_{I} \\
(\mu \mathrm{eV})\end{array}$ & $\begin{array}{c}\tau_{\mathrm{iv}} \\
(\mathrm{ps})\end{array}$ & $\begin{array}{c}\tau_{p} \\
(\mathrm{ps})\end{array}$ \\
\hline 75 & -2 & 0.010 & 1.1 & 8.8 & 8 & 12 & $\ldots$ & 0.6 & $\ldots$ \\
75 & 0 & 0.018 & 1.9 & 9.4 & 5 & 2 & 6.5 & 12 & 0.28 \\
75 & +1 & 0.021 & 1.7 & 6.1 & 3.5 & 1 & 9 & 45 & 0.22 \\
300 & 0 & 0.03 & 1.2 & 1.4 & 1.2 & 2 & 13 & 4 & 0.40 \\
\hline \hline
\end{tabular}

lifetimes of $12 \mathrm{~ns}$ in monolayer graphene [40]. Therefore, the spin relaxation length becomes comparable to the device size, and uncertainties such as the spin lifetime in the adjacent uncovered BLG regions can affect the analysis. Moreover, it is not clear whether the spin relaxation follows purely the Dyakonov-Perel mechanism and if other sources of spin-orbit coupling become relevant for limiting $\tau_{\|}$and $\tau_{\perp}$ in BLG [41-43].

Lastly, we discuss the temperature dependence of the spin-lifetime anisotropy. The carrier density dependence of $\tau_{\perp} / \tau_{\|}$at $T=5 \mathrm{~K}$ is discussed in the Supplemental Material [29] and gives comparable results to $T=75 \mathrm{~K}$ $\left(\tau_{\perp} / \tau_{\|}=2\right.$ at $6 \times 10^{12} \mathrm{~cm}^{-2}$ and $\tau_{\perp} / \tau_{\|}=8$ at the CNP). Figure 4(a) shows the ratio $R_{\mathrm{nl} \beta} / R_{\mathrm{n} 10}$ measured at an angle of $\beta=45^{\circ}$ and zero back gate voltage $\left(n=4 \times 10^{11} \mathrm{~cm}^{-2}\right.$ measured at 5 and $75 \mathrm{~K}$ ). We observe a continuous decrease of $R_{\mathrm{nl} \beta} / R_{\mathrm{nl0}}$ as the temperature increases. At room temperature, $R_{\mathrm{nl} \beta} / R_{\mathrm{nl} 0}$ is close to 0.5 , which corresponds to an isotropic system where $\tau_{\perp} / \tau_{\|} \approx 1$. The full angular dependence of $R_{\mathrm{nl} \beta} / R_{\mathrm{nl} 0}$ at $T=300 \mathrm{~K}$ is shown in Fig. 4(b). We extract here $\tau_{\perp} / \tau_{\|}=1.2$, where we estimate the error margin to be between 1 and 1.4. Because of an increased gate leakage current, we are unable to reach the CNP at $300 \mathrm{~K}$. Therefore, we assume that the doping of the BLG flake remains constant over the measured temperature range, and, consequently, the carrier concentration at room temperature is $4 \times 10^{11} \mathrm{~cm}^{-2}$. We calculate $\tau_{p} \approx 0.4 \mathrm{ps} \sim$ $\tau_{\text {iv }} / 10$ indicating that the decrease of anisotropy at $300 \mathrm{~K}$ is caused by the decrease of $\tau_{\text {iv }}$. Note that the thermal broadening at $300 \mathrm{~K}$ causes a sizable spread in momenta that can lead to lower lifetime anisotropies because $\lambda_{I}$ diminishes fast with increasing $n$.

Theoretical calculations predict in contrast to our results a maximum of the anisotropy around $175 \mathrm{~K}$ [18]. Additionally, the anisotropy is predicted to be below 1 at low temperatures due to the suppression of intervalley scattering induced by electron-phonon interaction. Both predictions are not consistent with our observations, which
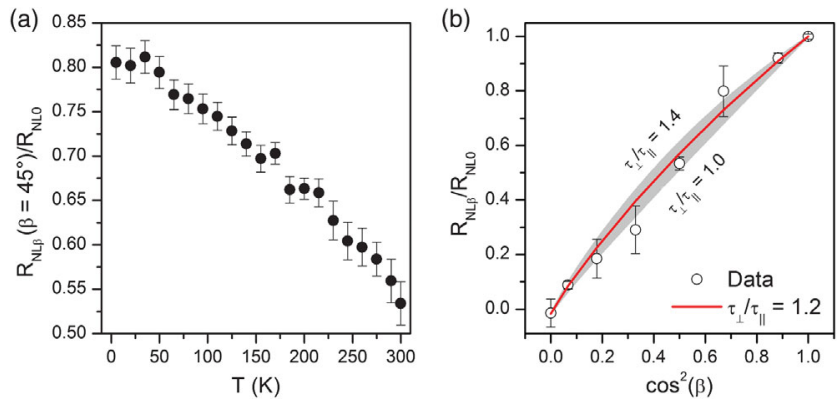

FIG. 4. (a) Temperature dependence of the ratio $R_{\mathrm{n} 1 \beta} / R_{\mathrm{n} 10}$ measured at $\beta=45^{\circ}$. The trend towards $R_{\mathrm{nl} \beta} / R_{\mathrm{n} 10}=0.5$ with increasing temperature implies that the anisotropy decreases. (b) Extraction of the $\tau_{\perp} / \tau_{\|}$for $T=300 \mathrm{~K}$ analogous to Fig. 2. We conclude that $\tau_{\perp} \sim \tau_{\|}$at room temperature. 
we attribute to two main differences between theory and experiment. First, the calculations are performed at $n=3 \times 10^{12} \mathrm{~cm}^{-2}$, which is significantly above $n$ for our device. As we have demonstrated in this Letter, the anisotropy is strongly affected by $V_{\mathrm{BG}}$, which changes the spin texture and the carrier density. Second, our device is fully encapsulated in $h$-BN, which can affect the phonon modes in BLG. At room temperature, these calculations predict $\tau_{\perp} / \tau_{\|}$above 50 with $\tau_{\|}$greater than $10 \mathrm{~ns}$, whereas we find an almost isotropic system and $\tau_{\|}=1.2 \mathrm{~ns}$.

In summary, we have studied the spin-lifetime anisotropy in BLG by oblique spin precession. $\tau_{\perp}$ is found to be up to 8 times larger than $\tau_{\|}$at the CNP. The anisotropy is found to decrease with increasing carrier concentration. An increase in the temperature above $75 \mathrm{~K}$ causes a decrease of $\tau_{\perp} / \tau_{\|}$, and around room temperature $\tau_{\perp}$ approaches a similar value as $\tau_{\|}$, implying that BLG becomes isotropic. We attribute this to the intrinsic outof-plane spin-orbit fields in BLG, which, despite their small magnitude, induce a significant spin-valley coupling that can be used to control spins in BLG $[11,18]$.

The authors acknowledge fruitful discussions with M. Gmitra, A. A. Kaverzin, and K. Zollner. This project has received funding from the European Unions Horizon 2020 research and innovation program under Grant No. 696656 and 785219 (Graphene Flagship Core 1 and Core 2), the Marie Curie initial training network Spinograph (Grant No. 607904), the DFG SFB 1277 (Projects No. A09 and No. B07), and the Spinoza Prize (for B. J. v. W.) by the Netherlands Organization for Scientific Research.

Note added.-Recently, a related work was uploaded on arXiv [44]

"These authors contributed equally to this work. j.c.leutenantsmeyer@rug.nl

[1] D. Xiao, G.-B. Liu, W. Feng, X. Xu, and W. Yao, Phys. Rev. Lett. 108, 196802 (2012).

[2] J. R. Schaibley, H. Yu, G. Clark, P. Rivera, J. S. Ross, K. L. Seyler, W. Yao, and X. Xu, Nat. Rev. Mater. 1, 16055 (2016).

[3] Y. K. Luo, J. Xu, T. Zhu, G. Wu, E. Joan, W. Zhan, M. R. Neupane, and R. K. Kawakami, Nano Lett. 17, 3877 (2017).

[4] A. Avsar, D. Unuchek, J. Liu, O. L. Sanchez, K. Watanabe, T. Taniguchi, B. Özyilmaz, and A. Kis, ACS Nano 11, 11678 (2017).

[5] A. W. Cummings, J. H. García, J. Fabian, and S. Roche, Phys. Rev. Lett. 119, 206601 (2017).

[6] T. S. Ghiasi, J. Ingla-Aynés, A. A. Kaverzin, and B. J. van Wees, Nano Lett. 17, 7528 (2017).

[7] L. A. Benítez, J. F. Sierra, W. S. Torres, A. Arrighi, F. Bonell, M. V. Costache, and S. O. Valenzuela, Nat. Phys. 14, 303 (2018).

[8] Z. Wang, D.-K. Ki, H. Chen, H. Berger, A. H. MacDonald, and A. F. Morpurgo, Nat. Commun. 6, 8339 (2015).
[9] Z. Wang, D. K. Ki, J. Y. Khoo, D. Mauro, H. Berger, L. S. Levitov, and A.F. Morpurgo, Phys. Rev. X 6, 041020 (2016).

[10] S. Zihlmann, A. W. Cummings, J. H. Garcia, M. Kedves, K. Watanabe, T. Taniguchi, C. Schönenberger, and P. Makk, Phys. Rev. B 97, 075434 (2018).

[11] S. Konschuh, M. Gmitra, D. Kochan, and J. Fabian, Phys. Rev. B 85, 115423 (2012).

[12] M. Gmitra, K. Zollner, and J. Fabian (to be published).

[13] J. Martin, N. Akerman, G. Ulbricht, T. Lohmann, J. H. Smet, K. Von Klitzing, and A. Yacoby, Nat. Phys. 4, 144 (2008).

[14] J. C. Leutenantsmeyer, A. A. Kaverzin, M. Wojtaszek, and B. J. van Wees, 2D Mater. 4, 014001 (2017).

[15] D. Van Tuan, S. Adam, and S. Roche, Phys. Rev. B 94, 041405 (2016).

[16] E. I. Rashba, Phys. Rev. B 79, 161409 (2009).

[17] M. H. D. Guimarães, P. J. Zomer, J. Ingla-Aynés, J. C. Brant, N. Tombros, and B. J. van Wees, Phys. Rev. Lett. 113, 086602 (2014).

[18] L. Wang and M. W. Wu, Phys. Rev. B 87, 205416 (2013).

[19] N. Tombros, S. Tanabe, A. Veligura, C. Jozsa, M. Popinciuc, H. T. Jonkman, and B. J. van Wees, Phys. Rev. Lett. 101, 046601 (2008).

[20] B. Raes, J. E. Scheerder, M. V. Costache, F. Bonell, J. F. Sierra, J. Cuppens, J. van de Vondel, and S. O. Valenzuela, Nat. Commun. 7, 11444 (2016).

[21] S. Ringer, S. Hartl, M. Rosenauer, T. Völkl, M. Kadur, F. Hopperdietzel, D. Weiss, and J. Eroms, Phys. Rev. B 97, 205439 (2018).

[22] W. Han and R. K. Kawakami, Phys. Rev. Lett. 107, 047207 (2011).

[23] T. Y. Yang, J. Balakrishnan, F. Volmer, A. Avsar, M. Jaiswal, J. Samm, S. R. Ali, A. Pachoud, M. Zeng, M. Popinciuc, G. Güntherodt, B. Beschoten, and B. Özyilmaz, Phys. Rev. Lett. 107, 047206 (2011).

[24] A. Avsar, T. Y. Yang, S. Bae, J. Balakrishnan, F. Volmer, M. Jaiswal, Z. Yi, S. R. Ali, G. Güntherodt, B. H. Hong, B. Beschoten, and B. Özyilmaz, Nano Lett. 11, 2363 (2011).

[25] I. Neumann, J. Van De Vondel, G. Bridoux, M. V. Costache, F. Alzina, C. M. S. Torres, and S. O. Valenzuela, Small 9, 156 (2013).

[26] J. Ingla-Aynés, M. H. D. Guimarães, R. J. Meijerink, P. J. Zomer, and B. J. van Wees, Phys. Rev. B 92, 201410 (2015).

[27] A. Avsar, I. J. Vera-Marun, J. Y. Tan, G. K. W. Koon, K. Watanabe, T. Taniguchi, S. Adam, and B. Özyilmaz, NPG Asia Mater. 8, e274 (2016).

[28] P. J. Zomer, M. H. D. Guimarães, J. C. Brant, N. Tombros, and B. J. van Wees, Appl. Phys. Lett. 105, 013101 (2014).

[29] See Supplemental Material at http://link.aps.org/ supplemental/10.1103/PhysRevLett.121.127702 for details on the fabrication, the characterization of the device, the estimation of the electric field, and the analysis and discussion of additional measurements, which includes Refs. [30,31].

[30] J. Fabian, A. Matos-Abiague, C. Ertler, P. Stano, and I. Zutic, Acta Phys. Slovaca 57, 342 (2007).

[31] E. C. Stoner and E. P. Wohlfarth, Phil. Trans. R. Soc. A 240, 599 (1948). 
[32] E. V. Castro, K. S. Novoselov, S. V. Morozov, N. M. R. Peres, J. M. B. Lopes dos Santos, J. Nilsson, F. Guinea, A. K. Geim, and A. H. Castro Neto, Phys. Rev. Lett. 99, 216802 (2007).

[33] J. B. Oostinga, H. B. Heersche, X. Liu, A. F. Morpurgo, and L. M. K. Vandersypen, Nat. Mater. 7, 151 (2008).

[34] Y. Zhang, T.-T. Tang, C. Girit, Z. Hao, M. C. Martin, A. Zettl, M. F. Crommie, Y. Ron Shen, and F. Wang, Nature (London) 459, 820 (2009).

[35] M. Gurram, S. Omar, and B. J. van Wees, Nat. Commun. 8, 248 (2017).

[36] M. Gurram, S. Omar, and B. J. van Wees, 2D Mater. 5, 032004 (2018).

[37] B. Raes, A. W. Cummings, F. Bonell, M. V. Costache, J. F. Sierra, S. Roche, and S. O. Valenzuela, Phys. Rev. B 95, 085403 (2017).
[38] T. Maassen, I. J. Vera-Marun, M. H. D. Guimarães, and B. J. van Wees, Phys. Rev. B 86, 235408 (2012).

[39] T. Zhu and R. K. Kawakami, Phys. Rev. B 97, 144413 (2018).

[40] M. Drögeler, C. Franzen, F. Volmer, T. Pohlmann, L. Banszerus, M. Wolter, K. Watanabe, T. Taniguchi, C. Stampfer, and B. Beschoten, Nano Lett. 16, 3533 (2016).

[41] D. Kochan, S. Irmer, M. Gmitra, and J. Fabian, Phys. Rev. Lett. 115, 196601 (2015).

[42] D. Van Tuan, F. Ortmann, D. Soriano, S. O. Valenzuela, and S. Roche, Nat. Phys. 10, 857 (2014).

[43] W. Han, R. K. Kawakami, M. Gmitra, and J. Fabian, Nat. Nanotechnol. 9, 794 (2014).

[44] J. Xu, T. Zhu, Y. K. Luo, Y.-M. Lu, and R. K. Kawakami, following Letter, Phys. Rev. Lett. 121, 127703 (2018). 